\section{La prisionización y los conflictos en el entorno penitenciario: investigación documental}

Imprisonment and conflicts in the prison environment: documentary research

\section{RESUMEN}

The diverse experiences in life allow us to face situations that test our ability to adapt to the environment; we are social beings who appropriate and develop certain skills to join any of the socializing institutions we relate to. Therefore, conflict can be understood as inherent to human development, the psychosocial skills, or tools that we have, contribute to facing problematic situations, in the deliberation of decision making, tolerance, conflict management, search for agreements, and consensus to solve disputes in any area of our life. The above makes us understand that in all the areas in which we work, including the prison system, the poor approach to conflicts prevails, since inadequate decision making encourages the aggressive environment characteristic of the prison environment and the process of incarceration. By making the series of conflicts and violence in the prison system visible, we will be able to identify the causes and consequences they generate in the prison population. The premise is not to put an end to conflicts, nor to get used to them, but to strengthen conflict management and achieve peaceful solutions through dialogue and the establishment of agreements.

Palabras clave: Imprisonment; Conflict; Prison; Prison; Violence; Mediation

\section{ABSTRACT}

We are social beings which we appropriate and develop certain skills to incorporate ourselves into any of the socializing institutions that we relate to. That is why, the conflict can be understood as inherent to human development, the skills or psychosocial tools that are available, contribute to cope with problematic situations, in the deliberation of decisionmaking, tolerance, management of conflicts, search for agreements and consensus to solve controversies in any area of our life. The foregoing makes us understand that in all the areas that we perform, including the prison system, the wrong approach to conflicts prevails, since inadequate decision-making encourages an aggressive environment, which is characteristic of the prison environment and the process of imprisonment. The experiences of the constant riots in the various prisons of the country have shown us the need to manage conflicts and provide a conciliatory approach to disputes, meaning learning to live with conflicts and being able to manage them. By making visible the series of conflicts and violence in the prison system, we will be able to identify the causes and consequences that they generate in the population deprived of liberty. The premise is not to end conflicts or get used to them, but rather to strengthen conflict management and achieve pacifying solutions through dialogue and establishing agreements.

Keywords: Imprisonment; Conflict; Prison; Violence; Mediation
RELIGACIÓN

REVISTA DE CIENCIAS SOCIALES Y HUMANIDADES JOURNAL OF SOCIAL SCIENCES AND HUMANITIES
REVISTA DE CENCIAS SOCIAIS E HUMANAS

INFORMACIÓN:

https://doi.org/10.46652/rgn.v6izo.848 ISSN $2477-9083$

Vol. 6 No. 30, 2021. e210848

Quito, Ecuador

Enviado: septiembre 30, 2021 Aceptado: noviembre 24, 2021 Publicado: diciembre 11, 2021

Publicación Continua

Sección Dossier | Peer Reviewed
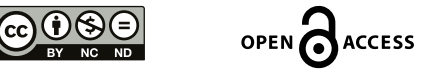

AUTORA:

(D) Martha Vanesa Díaz Padilla Instituto de Ciencias Psicojurídicas y Sociales - México

vanelucas@yahoo.com.mx

\section{Conflicto de intereses}

La autora declara que no existe conflicto de interés posible.

Financiamiento

Existió asistencia financiera por parte de la Asociación Mexicana en Psicología Jurídica y Derecho Penal. El apoyo consistió en especie y en el proceso de investigación.

\section{Agradecimiento}

Asociación Mexicana en Psicología Jurídica y Derecho Penal y a su equipo:

Dra. Patricia Olivia González Lozano. Dra. Luz Anyela Morales Quintero. Dr. Eric García López.

Nota

El presente artículo es parte de las lineas de investigación del Instituto de Ciencias Psicojurídicas y Sociales IPSIJUS y la Asociación Mexicana en Psicología Jurídica y Derecho Penal

ENTIDAD EDITORA 


\section{Introducción}

Toda trasgresión de la norma tiene repercusiones sociales y de impacto; además de los costos en la investigación policial y de procesamiento de los delitos, por mencionar algunos. Sin embargo, las consecuencias van más allá del impacto en el sistema de justicia y debido proceso, ya que, se presentan consecuencias de efecto paralelo victima-victimario. Datos que se publicaron en la Encuesta Nacional de Victimización y Percepción sobre Seguridad Pública (2021), realizada por el Instituto Nacional de Estadística y Geografía, menciona que, en 2020, a nivel nacional, se estiman 21.2 millones de víctimas de 18 años y más, lo cual representa una tasa de 23520 víctimas por cada cien mil habitantes, cifra estadísticamente inferior a la estimada en 2019 que fue de 24849 . Estos datos inciden directamente en la implementación o en el fallo de políticas públicas. Mientras que con las personas privadas de libertad podemos mencionar que el costo social y económico de la reinserción social es un tema pendiente en muchas de las agendas gubernamentales, puesto que, el sistema penitenciario no es considerado un tema relevante mucho menos prioritario. Ya que, los centros de reclusión en México no han alcanzado el desarrollo total de una metodología y técnicas necesarias para una adecuada reinserción social de las personas privadas de libertad. Al interior de estos lugares, lo único que, medianamente, se alcanza es la contención de la población; las riñas, las pugnas al interior, los robos, los desacuerdos y demás situaciones divergentes se hacen presente todos los días, lo que genera confrontaciones directas con consecuencias devastadoras. Tal como lo refiere el Diagnóstico Nacional de Supervisión Penitenciaria (2020), realizado por la Comisión Nacional de Derechos Humanos, donde se menciona las irregularidades con mayor incidencia detectadas en los centros de reclusión.

Para investigar el tema de conflictos en reclusión se debe considerar que el sistema penitenciario y, de forma puntual, los centros de reinserción, ejecutan - justificada legalmente-, violencia de forma directa y estructural; dirigida hacia las personas privadas de libertad, así como al personal penitenciario; ambos grupos de población experimentan diversas situaciones conductuales como adaptación al entorno controlado, las cuales son muy similares entre sí, a lo que se denomina prisionización. Es preciso enfatizar que el sistema penitenciario siempre "domina" o "controla" las actividades de aquellos privados de libertad, desde la comida, el vestido, la clasificación, con quién socializan, entre otros, las personas se convierten en un número más. Algo similar sucede con los operadores penitenciarios, ya que, en ocasiones recurren a licencias médicas para justificar el ausentismo, presentando distintas manifestaciones cognitivas que influyen directamente en su salud mental y permean su desarrollo profesional. Considerando que el personal penitenciario es insuficiente para las grandes cifras de personas privadas de libertad. Para brindar atención, labores de clasificación, seguridad y ofrecer un tratamiento individualizado se contaba al cierre de 2020 con reporte de 39501 personas, de las cuales, 62.4\% fueron hombres y $37.6 \%$ mujeres. Referente al personal adscrito a los centros penitenciarios federales, $44.9 \%$ fueron hombres y $55.1 \%$ mujeres. Si bien, habrá que considerar la situación de emergencia sanitaria de COVID-19 la cual, restringió el ingreso y visitas a los centros de reclusión, el sistema penitenciario funcionó de forma intermitente y pausada, debido al aislamiento de algunos operadores penitenciarios. 
Desde la práctica hemos constatado que para esta población hablar de salud mental ha resultado un tabú, no se ha visibilizado en la catedra, investigación y en los contextos institucionales que tengan injerencia en los entornos controlados. Así, se puede decir que existe una falla en el sistema penitenciario, ya que no procura la salud mental del personal y de las personas privadas de libertad; toda vez que desde diversas perspectivas el entorno penitenciario se caracteriza por manifestaciones de la violencia estructural y el casi inexistente tratamiento de higiene mental. El proceso de prisionización facilita que ambos grupos (personal penitenciario y personas privadas de libertad), se involucren en subgrupos para favorecer o reforzar su necesidad de valía y logro, mediante un clima de hostilidad, en consecuencia, las confrontaciones pueden culminar en riñas, así como en abuso de autoridad. Romero (2019) define la prisionización como la asimilación e internalización de la subcultura carcelaria por parte del sujeto, que no sólo supone la aceptación de normas y códigos institucionales (de la cárcel como institución), sino, sobre todo, de las propias reglas y jerarquías entre los internos. Tal como lo refleja el autogobierno o cogobierno, dato que se visibiliza en el Diagnóstico Nacional de Supervisión Penitenciaria (2020), realizado por la Comisión Nacional de Derechos Humanos, actividad que se presentó en 15 de los 113 centros penitenciarios supervisados donde las personas privadas de la libertad realizan o participan en actividades propias de la autoridad, tal como, el lenguaje, vestimenta, horarios, actividades, visita, entre muchos más, como parte de la subcultura penitenciaria. De esta manera, la prisionización obedece más a una aceptación del encierro, que, a una cuestión de temporalidad,

Por consiguiente, es necesario ponderar la importancia de influir en la emoción mediante habilidades psicoemocionales y técnicas psicoeducativas que permitan desarrollar la búsqueda de soluciones, donde no impere una respuesta violenta. Pesqueira (2015), a través de investigaciones realizadas sobre el binomio, emociones y violencia, comenta que tener conocimiento de las emociones propias y ajenas, puede favorecer la capacidad de comprender, describir y predecir los estados mentales por los que atraviesa una persona, por ende, la capacitación en el manejo de emociones y conflictos debe prevalecer la voluntariedad, ya que, se podrá contar con aquellas personas que tengan iniciativa para gestionar los conflictos y perfilarse hacia aquellas que ejerzan un liderazgo positivo dentro de su grupo. Por lo que el desarrollo de habilidades sociales es prioritario, específicamente, en el manejo de emociones y en la comprensión del por qué un sentimiento contribuirá en generar nuevos aprendizajes con las diversas afecciones, como lo son: ansiedad, enojo, tristeza o alegría, lo que en consecuencia hacer racional la causa y el efecto de cada una de las conductas. Dentro del sistema penitenciario se esperaría, que cada persona privada de libertad trabaje desde el desarrollo de habilidades sociales para comprender el impacto de su actuar, así como, la toma de decisiones ante una situación particular. Sin embargo, como sociedad, se carece de una cultura de prevención de conductas conflictivas, de promoción una comunicación asertiva y de fomentación de mecanismos que favorezcan la solución de disentimientos, promoviendo el diálogo. Ya que, en diversas ocasiones la tendencia a la gratificación inmediata desde posturas emocionales ha restado importancia a la generación de alternativas viables ante las controversias, dejando de lado la premisa de que las personas privadas acepten las consecuencias y responsabilidades de su proceder, modificando su conducta y contribuir a la toma de decisiones durante su internamiento, de estar manera se puede atender 
de forma prioritaria, el diálogo propositivo, sin generar violencia ni la sumisión impuesta por la coacción unilateral que es característico del sistema penitenciario. Díaz (2018) comenta que los centros de reclusión mexicanos no han alcanzado dentro de sus labores, el desarrollo total y adecuado de una metodología y técnica necesarias con vías de la reinserción social de las personas sujetas a la privación de su libertad, en el sentido, que no se ha podido desarrollar una política de prevención de delitos dirigida a la vida interior de los centros penitenciarios, un tema relevante es la sobrepoblación penitenciaria, tal como se mencionó anteriormente el poco recurso humano, material y de eficacia en el plan de actividades. Sustentado en las cifras del Instituto Nacional de Estadística y Geografía (INEGI), reportando al cierre de 2020, la cifra de personas privadas de la libertad a nivel nacional fue de 211 169, de las cuales, 92.3\% (194 841) se registró en los centros penitenciarios estatales y $7.7 \%$ (16 328) en los centros penitenciarios federales. Del total nacional, 94.4\% fueron hombres y 5.6\% mujeres. Comparado con 2019, se registró un aumento de $6.2 \%$ en 2020. Lo cual resulta considerable bajo situación de emergencia sanitaria de COVID-19.

Por tal razón, se pone de manifiesto una de las limitaciones del sistema penitenciario es el factor humano, ya sea por cuestiones presupuestales o de vocación del personal profesional, considerando que uno de los factores de riesgo a tomar en cuenta en la dinámica de motines y correctivos disciplinarios es la falta de "gobernabilidad" del personal de seguridad y custodia. Como se menciona en el DNSP (2020). Al mencionar que las riñas se agravan y conjuntan con el hacinamiento, inadecuadas instalaciones, falta de personal y el profesionalismo de éstos, la delegación de actividades de autoridad a las personas privadas de la libertad, el nivel de vigilancia, la insuficiencia de servicios básicos como la mediación para la solución de conflictos interpersonales derivados de la convivencia, condiciones deplorables de trabajo, enfrentamiento de grupos de poder, libre circulación de dinero, concesiones de privilegios, anarquía, entre otros, como el efecto pandémico en todas las esferas. En consecuencia, resulta necesaria y por demás indispensable la restructuración de intervenciones que pudiera realizar el personal penitenciario con enfoque en solución de conflictos.

\section{Metodología}

El presente trabajo, se realizó considerando el enfoque exploratorio descriptivo, ya que, su principal propósito, fue definir eventos y algunas situaciones representativas durante el proceso de prisionización y la relación de esta con los conflictos en el ambiente penitenciario. El tema de prisionización y conflictos penitenciarios ha resultado poco abordado en la investigación, situación que justifica el tipo de investigación implementada. Se exploró un panorama general sobre las situaciones representativas en la documentación del conflicto en el sistema penitenciario. Para ello se estableció una búsqueda en diversas bases de datos las cuales son de libre acceso, con la finalidad de realizar investigación exploratoria, algunas de estas bases de datos son Scopus, Redalyc, Dialnet, Science Direct y catálogos de la UNAM.

Tal como lo refiere Ávila (2006). Un trabajo innovador requiere contar con estudios e información previa. Para ello se consideró, visualizar las bondades y alcances de la gestión de conflictos mediante el diálogo propositivo. Dejando entreabierta la posibilidad de que posteriormente se profundice sobre los alcances y resultados que brinda la mediación penitenciaria con personas privadas de libertad. 


\section{Desarrollo}

\subsection{Conflicto y violencia}

A partir de diferentes enfoques y cuestionamientos epistemológicos existe una diversidad de definiciones sobre el conflicto; en este trabajo se conceptualizará el mismo desde múltiples posturas. De acuerdo con Ausín (2005) la palabra conflicto proviene del latín conflictus, derivado de confligere, que significa pelear, combatir, disputar. Es importante comprender que el conflicto puede presentarse de diversas maneras, y que estas pueden coincidir en una o más personas. En términos concretos, se puede delimitar el conflicto a una situación de lucha o desacuerdo que genera diversas reacciones emocionales, las cuales inciden directamente en la toma de decisiones y en el resultado de alguna controversia.

Parra et al.,(2014) definen el conflicto como aquellas situaciones en las que se presentan diferencias de intereses, valores o necesidades entre las personas, grupos, organizaciones o estados que dificultan la resolución de un problema. Ante tal escenario, la percepción y las definiciones tienen un valor agregado; podríamos ejemplificarlo, metafóricamente, con un caleidoscopio, en el cual se puede percibir, desde diferentes enfoques, los pequeños fractales que contiene en su interior y desde la misma mirilla puede percibirse un fondo diverso - el conflicto visto desde diferentes posturas-; si giramos un poco el caleidoscopio, podremos visibilizar distintas perspectivas.

Es así como, el conflicto requiere ser abordado desde diferentes esferas, denotando que la génesis de este incide en diversos factores, uno de ellos son las emociones, las que se deberán de trabajar y abordar desde técnicas psicoeducativas, que coadyuven a su óptimo manejo; tal como lo refiere Huertas (2011) la conciencia de las propias emociones es el eslabón que une la inteligencia emocional. En este sentido las emociones son la base de la estructura de un conflicto y sin el trabajo en ello, permean la toma de decisiones y obstaculizan visibilizar las posibles alternativas.

Uno de los autores más actuales y que ha contribuido a la implementación de varios programas que favorecen el diálogo. Ríos et al., (2016) han descrito la naturaleza y los niveles de violencia en reclusión de la siguiente manera:

Violencia Estructural: es aquella que proviene principalmente del sistema o ente en el cual se rige. La estructura institucional responde con violencia.

Respuesta Penal: se caracteriza por el funcionamiento de la maquinaria del sistema penal. En este rubro, la violencia que se utiliza es selectiva, ya que se ejecuta de acuerdo con las circunstancias, para ciertos delitos se utiliza la violencia directa y dinámica considerando el sufrimiento, el coste económico y la gravedad de algunos delitos. Ríos et al., (2016) mencionan que la respuesta penal termina por dividir y enfrentar lo que debería integrar, no es de extrañar que algún penalista diga que, aun cuando está rodeado de límites y garantías, el derecho penal posee una intrínseca brutalidad que nos permite dudar de su moralidad.

El Primer Grado: aquí la institución ejerce el mayor grado de violencia sobre las personas privadas de libertad, justificado por su comportamiento y una posible trasgresión de las normas internas. Las medidas son consideradas como correctivos disciplinarios, también 
llamados segregaciones, con ello se da un proceso de re-etiquetamiento, ya que, se trata a las personas privadas de libertad como, "alto riesgo" para el resto de la población y/o trabajadores. Las dinámicas interpersonales se vuelven más violentas y estigmatizantes, porque favorecen la realización de actos violentos de forma recurrente entre el recluso y las autoridades, tornándose en un ciclo de violencia ascendente que propicia el aumento de la conflictividad y puede impactar de forma directa en el resto de la población penitenciaria.

Control directo: en este rubro la violencia se manifiesta más claramente, se agudizan diversas confrontaciones. Por lo tanto, los conflictos son recurrentes y manifiestos.

A lo largo de la historia, los conflictos incentivaron la generación de cambios en pro de la sociedad; sin embargo, también están aquellos casos en los que un conflicto se convirtió en violencia, guerra y confrontaciones constantes. Johan Galtung (1998) lo denominó meta conflicto, comprendido como el conflicto que emana, o surge tras, el conflicto de raíz, la capa superior. Siendo consecuencia de diversas manifestaciones violentas, las cuales, al no ser abordadas, corren el riesgo de escalar hacia conductas agresivas y desafiantes, generando un clima de violencia. El conflicto tiene una raíz, pero lo que marca la diferencia en la tipología de este son las diversas variables que se encuentran presentes y que contribuyen a las manifestaciones de la confrontación, como el caso de ambiente en prisión, el cual es característico de hostilidad y diversas manifestaciones de violencia.

Coincidiendo con Redorta (2007), quien refiere que las controversias se pueden desarrollar de diferentes formas, sin embargo, lo relevante son los procesos de adaptación en los cuales se presenta y desarrolla el propio conflicto. $Y$ es así como, hablando del conflicto en el sistema penitenciario, podemos precisar que dichas manifestaciones, se encuentran en la fase de escalada crónica y habitual, ante las diversas manifestaciones de violencia y el clima hostil es necesario generar mecanismos de adaptación en el entorno adverso e hipervigilante que se impone a las personas privadas de libertad clasificados como, "problemáticos". De alguna forma podemos comprender que las exteriorizaciones de violencia, por las personas privadas de libertad, son una respuesta de sobrevivencia, tal como lo expresan las leyes naturales, lo cual es parte del proceso de prisionización. La violencia está íntimamente ligada al conflicto, puede ser un actuar derivado de este aun cuando en la prevalencia existan manifestaciones diversas, cuando las confrontaciones escalan y se agudizan, pueden convertirse en uno de los muchos caminos que toma el conflicto.

Osorio (2012) establece una diferencia entre el conflicto y la violencia. Define conflicto como una situación en la que al menos un autor - persona, comunidad, estado, (del mismo o diferente rango), lo que conduce a una oposición, enfrentamiento o lucha. Así, podemos comprender que el conflicto y sus diversas manifestaciones pueden volverse crónicas, y, de no abordarse, se incrementa la presencia de confrontaciones directas e indirectas.

Osorio (2012) describe la violencia y la correlaciona con las manifestaciones de conflicto, la define como, 
...aquellos fenómenos o actos personales y/o sociales en los que se aplica un exceso de fuerza para la consecución de un fin o las realizaciones efectivas de algunos de los actores en el juego social quedan por debajo de sus realizaciones potenciales, es decir, cuando lo potencial sea mayor que o efectivo y esto sea socialmente evitable (p. 56).

Es ahí donde radica la complejidad del conflicto y la exacerbación de este, lo cual incide directamente en la posibilidad de gestionarlo.

Habrá que comprender que el conflicto es un proceso de interacción a partir de un enfoque determinado en el que inciden directamente los intereses, las necesidades y las posturas ante una discrepancia en común; al abordarlo desde una postura unicausal es mantenerlo en un panorama reduccionista que limita las líneas de análisis, intervención y gestión de este. Es importante mencionar que, a partir de la forma en que se aborde el conflicto, podrá delimitarse si el resultado es transformativo, solución específica del conflicto, o un paliativo para disminuir la carga ansiógena entre los involucrados. Si se considera aproximar desde una perspectiva transformativa, habrá que tomar en cuenta que todo conflicto contiene una faceta positiva; se deberá promover un acercamiento constructivo y propositivo, enriquecido mediante el diálogo y la comunicación asertiva, en tal situación, la gestión de las emociones debe consolidarse mediante procesos de mediación, para desarrollar habilidades y aprender a regular la manifestación de dichas emociones.

\subsection{Manifestaciones de violencia como proceso de prisionización}

Entre las deficiencias conductuales podemos mencionar la intolerancia a la frustración, las respuestas violentas e impulsivas hacia la dinámica de convivencia y la poca capacidad para gestionar conflictos por la vía del diálogo. Es necesario resaltar que, el manejo y la gestión de emociones resulta primordial en el ambiente penitenciario, toda vez que, en una situación de encierro y entorno controlado, las personas privadas de libertad experimentan una serie de manifestaciones de violencia. Ríos et al., (2016) menciona algunas principalmente en el sistema penitenciario:

\footnotetext{
Violencia física: se entiende como todas aquellas manifestaciones confrontativas y directas que se utilizan de manera recurrente en el sistema penitenciario, las cuales generan mayores controversias de forma directa, a su vez inciden directamente en la generación de diversas repercusiones aún más violentas.

Violencia psicológica: es un intento de destrucción mental que busca desestabilizar; cabe mencionar que es aplicable en todo el proceso de prisión y se manifiesta de forma directa e indirecta (manifestaciones sutiles).

Violencia estructural: comúnmente la ejecutan entes gubernamentales y aquellos organismos que regulan a los actores que están sujetos a sus requerimientos, lo anterior como parte de un control institucional.
}

Al hablar del sistema penitenciario, debemos considerar que éste fue diseñado por un sistema vindicativo, cuyo eje primordial es el "control" y el "aislamiento social" desde un enfoque sociológico del delito. La convivencia en estos centros genera un importante número de conflictos 
interpersonales, algunos de ellos desembocan en actos de indisciplina, en ocasiones, cuando la imposición de sanciones no resuelve el problema, la persona "castigada" se queda segregada, contribuyendo en generar hostilidad, agresión, confrontación y un clima de constante violencia. Ya lo dijo Martín Luther King en alguno de sus discursos, "la violencia crea más problemas que los que resuelve, y por tanto nunca conduce a la paz".

Si consideramos la postura ideológica de Neuman (2005), quien refiere que el objetivo del encierro no es recibir un castigo, sino ser castigado en forma diaria, continua y persecutoria, al poner un pie en la cárcel se comienza a cumplir la condena, aunque las leyes constitucionales, los códigos penales, procesales y de ejecución de la pena señalen exactamente lo contrario. Favoreciendo el proceso de prisionización. Es por ello indispensable comprender e identificar el conflicto en el ambiente penitenciario desde la experiencia de vida de cada una de las personas privadas de libertad, considerando sus estrategias psicosociales para hacerle frente. Para algunos autores, la controversia representa una posibilidad de aprendizaje; es el caso de Johan Galtung, quien menciona que un conflicto puede interpretarse como crisis y/o oportunidad y lo denomina "metaconflicto". El conflicto se ha abordado desde diversos enfoques, cada uno de los cuales ha dejado clara la necesidad de gestionarlo tomando en consideración sus múltiples causas y factores desencadenantes (como se citó en Calderón, 2009).

Para tener una visión integral sobre los conflictos en reclusión hay que considerar el proceso de prisionización el cual, se desarrolla íntimamente en la cotidianidad del sistema penitenciario y durante la adaptación a este, se presentan una serie de modificaciones conductuales, las cuales favorecen la dinámica de supervivencia en el entorno controlado. Las manifestaciones de esta prisionización también se presentan entre los operadores del sistema penitenciario, quienes adoptan diversas normas, reglas y actividades que contribuyen al desgaste profesional y la deshumanización. El ambiente penitenciario se caracteriza por ser un entorno violento, con agresiones constantes, sin embargo, es importante precisar que, en esta aportación documental, la prisionización se enfoca como una de las líneas paralelas en la génesis del conflicto en el entorno penitenciario, ya que diversos autores han referido que, la importancia y divergencia de la prisionización puede ser comprendida como un proceso de “adaptación” al entorno penitenciario. Como afirma Romero (2019) quien menciona que todas aquellas personas que se encuentran en el proceso de prisionización se ven obligadas a decidir entre tres aspectos que contribuirán a la consolidación del proceso:

1. Adherirse a la subcultura carcelaria o prisionización primaria.

2. Cumplir con la normativa institucional o prisionización secundaria.

3. Oscilar entre subcultura y normatividad, mediante estrategias que le indiquen cuándo responder a una u otra, con todos los costos y la desprotección que ello implica o prisionización terciaria.

En consecuencia, se interrelaciona estrechamente al proceso de prisionización como un dinamismo inherente a la estancia penitenciaria, aun cuando todos los sujetos tienen habilidades 
de afrontamiento. Por otra parte, conforme a las referencias antes citadas se concluye que toda persona privada de libertad termina asumiendo su situación de encierro en un ambiente controlado. La convivencia entre las personas privadas de libertad y el personal representa el parteaguas de esa dinámica de riesgo y violencia. Recordemos que el entorno controlado es característico del sistema penitenciario, el cual, tiene como objetivo confinar a quienes trasgreden la norma, habría que recordar a Hobbes (1994) cuando menciona que la finalidad del castigo no es la venganza y la descarga de la ira, si no, a través del ejemplo, corregir tanto al ofensor como a los demás.

Los correctivos severos deben infringirse por aquellos crímenes que resultan más peligrosos para el común de las personas, por ende, surgen una serie de cuestionamientos sobre los fines de la sanción y la función del sistema penitenciario, sobre algunos de los objetivos para los que fue diseñado. Hay que considerar que cada conflicto es diferente, tanto en la génesis, el desarrollo y las personas que encuentran inmersas; las manifestaciones de poder tienen una reacción en cadena, comprendiéndolas como subordinadas e interdependientes, tanto en los reclusos como en los operadores del sistema. Los conflictos entre pares en el ámbito penitenciario se caracterizan por la prevalencia de una molestia anticipada, confrontaciones verbales, físicas y, en muchas ocasiones, la segregación de las personas privadas de libertad involucradas en la controversia. Dado que muchas de las personas que cohabitan no disponen de las herramientas cognitivas para funcionar de manera adecuada y hacer frente a los conflictos, suelen comportarse de forma trasgresora. Una de las limitantes es la falta de autorregulación y de inhibición de respuestas violentas, lo cual hace que la gestión de las emociones sea deficiente. Se parte de la premisa de que diversos procesos de mediación podrían aprender a regular esas deficiencias conductuales mediante técnicas psicoeducativas y que las personas privadas de libertad puedan reconocerse en la otredad, con las mismas necesidades, malestares, sensibilidad a las condiciones y cualidades humanas. Aunque implique todas estas cosas, el conflicto se refiere también, a la interacción de las personas con otras como seres humanos. Al generar un conflicto, sin ser abordado ni dialogado, coadyuva en ocasionar una serie de interacciones humanas de enojo introyectado y reprimido. La multifactoriedad del problema y perspectiva transformadora son una propuesta arriesgada y realista; no siempre habrá oportunidad de llegar a un punto de encuentro, pero siempre existe la posibilidad de entender y escuchar al otro.

Es necesario trabajar sobre las posturas de poder dentro de los procesos de solución de conflictos o mediación, ya que, de no hacerlo se está limitando el abordaje de estos y se fomenta la represión de las personas más débiles.

\section{Discusión}

Ciertos postulados de la creación del sistema penitenciario nos permiten comprender que la cárcel está diseñada para contener de forma momentánea a quienes han trasgredido la norma, sin considerar su historia, necesidades o circunstancias. Dicha situación favorece la confrontación directa e indirecta, ya que durante un proceso de adaptación un individuo puede adoptar ciertas conductas del entorno en el que se desarrolla, que no necesariamente favorecen el diálogo y la participación de las partes involucradas en un conflicto; pareciera que el sistema penitenciario 
justifica y normaliza la violencia, dado que, la sociedad al estar inmersa en la justicia vindicativa considera al aislamiento y la segregación como los principales recursos para hacer frente a diversas conductas que trasgreden la norma.

En este sentido, la mediación penitenciaria ofrece un método de resolución de conflictos entre internos catalogados como "incompatibles", ya sea, por el propio centro o por instituciones penitenciarias e internos con un expediente sancionador abierto, es decir, que les hayan sido impuesta una sanción por alguna falta cometida en el centro (Pastor Seller, 2012). En este sentido, la mediación brinda la posibilidad de no repetir ciertas conductas disruptivas, al poner en evidencia diferentes problemas que han sido la causa de motines y linchamientos en reclusión, lo que visibiliza la ausencia de una política penitenciaria, es decir: la insuficiencia de operadores, la precariedad de sus instalaciones, la corrupción, la falta de programas estructurados orientados hacia la reinserción social y las nulas estrategias para la solución de controversias. Sustentado en la Ley Nacional de Ejecución de Penas (2016).

Uno de los objetivos de la existencia de la prisión se relaciona con la justicia vindicativa, en la que aquellas personas que trasgreden la ley deben ir a una institución de "castigo", donde el entorno penitenciario tiene la finalidad de restringir el libre tránsito y generar una convivencia obligatoria entre personas con diferentes historias de vida y crimino génesis. En relación con la función de las normas sociales, Sober y Wilson mencionan que estas actúan para mantener a un grupo unido; van más allá de las relaciones parentales, evitando los conflictos y promoviendo que los individuos sean benévolos y generosos hacia todos los miembros de un grupo socialmente definido (como se citó en Gómez, 2019).

De acuerdo con Montoya (2020), la vulnerabilidad hace que seamos más susceptibles y que estemos más expuestos al daño y al dolor. Está condicionada por factores intrínsecos y extrínsecos que, en todo caso, parten de reconocer la fragilidad originaria del humano, asociada a elementos sociales y ambientales, que muestran su carácter cambiante de acuerdo con las relaciones, los contextos y las circunstancias que la acompañan. En este sentido, son posibles de ser intervenidos con acciones solidarias que promuevan en adelante la prevención, ayuden a sanar las heridas y a rebasar el dolor. En concordancia con la óptica de la cárcel se simboliza una institución con un ambiente conflictivo de tipo latente; las personas se ven en la necesidad de implementar habilidades conductuales y socio afectivas para hacer frente a las diferencias personales e incidir directamente en un ambiente hostil. En este contexto, la dinámica social de los subgrupos y la división entre las personas privadas de su libertad se encuentran marcadas por la corrupción por parte de los operadores del sistema penitenciario, de las propias personas privadas de libertad y de sus familiares, la cual puede manifestarse de múltiples maneras.

\section{Conclusiones}

Habrá que considerar que el conflicto normativo está íntimamente relacionado con la trasgresión de las reglas, cuya función principal es regular y mantener la estructura social; así, aquellas personas que se desvían de ese "control social" son considerados desviados o trasgresores de las reglas, sin considerar su historia de vida, recursos y redes sociales; por lo cual la mediación 
penitenciaria en México como alternativa ante la solución de conflictos penitenciarios enfrenta varios retos; uno de ellos es aplicar las técnicas psicoeducativas a personas que han trasgredido una o varias veces la norma y que presentan un conflicto normativo de tipo longitudinal. De hecho, varios teóricos coinciden en que es más fácil aprender a comportarse de una manera socialmente aceptable al estar en comunidad que dentro de un ambiente aislado y difícil como lo es el área de castigo de una prisión.

Hay que puntualizar que un factor que contribuye en el desarrollo de problemáticas intramuros es la poca intervención en la gestión de emociones y solución dialogada de conflictos, lo cual, está relacionada estrechamente con la génesis de conflictos ya que los tratos diferenciados en el entorno penitenciario favorecen las diversas manifestaciones de violencia y problemáticas intramuros, así como, las conductas de abuso de poder; por lo que las personas privadas de libertad no confían en las autoridades ni vislumbran otra posible vía para solucionar los conflictos entre pares que no sea a través de la violencia y el poder. Situación que deberá abordarse desde la óptica de las organizaciones civiles quienes tendrán un trabajo relevante para gestionar conflictos y promover la aplicación de mediación penitenciaria en México, mediante un enfoque transformador de conflictos deberá dejar a un lado la coacción y punición del sistema penitenciario, que solo contribuyen a fomentar la violencia y confrontación, en contraposición a las premisas sobre prevención de comportamientos violentos, como lo puede ser la mediación penitenciaria, la cual, tiene un enfoque de justicia social.

\section{Referencias}

Arteaga, D. G., \& Quebradas, A. (2010). Funciones ejecutivas y marcadores somáticos: apuestas, razón y emociones. El Hombre y la Máquina, (34), 115-129.

Ávila, B. (2006). Introducción a la metodología de la investigación Edición electrónica. Eumed.net

Ausín, T. (2005). Entre la Lógica y el Derecho. Paradojas y conflictos normativos. Editorial Plaza y Valdés.

Calderón, P. (2009). Teoría de Conflictos de Johan Galtung. Paz y Conflictos. (2), 74-75. https://doi. org/10.30827/revpaz.v2io.432

Comisión Nacional de Derechos Humanos. (2020). Diagnóstico Nacional de Supervisión Penitenciaria 2020. https://cutt.ly/cYhkrt3

Constitución Política de los Estados Unidos Mexicanos. (2016). México: Comisión Nacional de los Derechos Humanos. CNDH México. https://cutt.ly/iYhkuLF

De Haro, A. (2012). Antropología del conflicto. Reflexiones sobre el nuevo orden global. Convergencia Revista de Ciencias Sociales, (60), 177-204. https://convergencia.uaemex.mx/article/view/1074

Díaz, P., Maffioletti, C., y Puhl, M. (Compiladores). (2018). Psicología Jurídica, Derechos Humanos, y Derecho Penal. Ediciones de la U.

Fuquen, A. (2003). Los conflictos y las formas alternativas de resolución. Tabula Rasa, (1), 265-278. https:// doi.org/10.25058/20112742.198

Galtung, J. (1998). Tras la violencia, $3 R$ : reconstrucción, reconciliación, resolución Afrontando los efectos visibles e invisibles de la guerra y la violencia. Gernika Gogoratuz. 
García, A., Pérez, M. y Pérez, M. (2019). Mediación como Método de Solución de Conflictos. Editorial Tirant lo Blanch.

García, M. y Melendro, M. (2013). El ambiente en prisión: La atención recibida por las reclusas y las relaciones intramuros. Pedagogía Social. Revista Interuniversitaria, (22), 44-56. https://doi.org/10.7179/ PSRI_2013.22.04

Goffman, E. (2001). Internados: ensayos sobre la situación social de los enfermos mentales. Amorrortu Editores.

Gutiérrez, C. (2010). Justicia Restaurativa. Oxford University Press.

Hobbes, T. (1994). Leviatán o la materia, forma y poder de una república eclesiástica y civil. Fondo de Cultura Económica.

Huertas Díaz, O. (2012). Crimen, emociones y castigo. Revista Logos Ciencia \& Tecnología, 3(2), 170-171. https://doi.org/10.22335/rlct.v3i2.165

Instituto Nacional de Estadística, Geografía e Informática. (2020). Censo Nacional de Sistema Penitenciario Federal y Estatales 2021 https://cutt.ly/8YhllZK

Instituto Nacional de Estadística, Geografía e Informática. (2020). Encuesta Nacional de Victimización y Percepción sobre Seguridad Pública 2021 https://cutt.ly/NYhlzXh

Isler Soto, C. (2018). Thomas Hobbes y la función de los jueces. Revista de estudios histórico-jurídicos, (40), 407-421. https://dx.doi.org/10.4067/S0716-54552018000100407

Lederach, J. (1992). Enredos, pleitos y problemas: Una guía práctica para ayudar a resolver conflictos. Editorial Clara-Semilla.

Ley Nacional de Ejecución Penal 61/2016, de 16 de junio, Regular los medios para lograr la reinserción social. Diario Oficial de la Federación, 16 de junio 2016. https://cutt.ly/AYhIE14

Ley Nacional de Mecanismos Alternativos de Solución de Controversias en Materia Penal. 2014, 29 de diciembre. Soluciones Alternas previstas en la legislación procedimental penal aplicable. Diario Oficial de la Federación. 29 de diciembre 2014. https://cutt.ly/eYhlUwb

López, M. (2010). El perfil del mediador. En E. García-López, Fundamentos de Psicología Jurídica y Forense. (pp. 373-395). Oxford University Press.

Montoya-Sánchez, M., Salinas-Arango, N., \& Puerta Lopera, I. (2021). Mediación transformativa: la vulnerabilidad como apertura a la transformación de las relaciones familiares. Opinión Jurídica, (42), 297-319. https://doi.org/10.22395/ojum.v2on42a12

Neuman, E. (2005). La prisión como control social en el neoliberalismo. Universidad Nacional Autónoma de México.

Osorio, S. (2012). Conflicto, Violencia y Paz: Un acercamiento científico, filosófico y bioético. Revista Latinoamericana de Bioética, 12(23), 52-69. https://doi.org/10.18359/rlbi.960

Parra, E., Darío, T., Franco, J. y Vallejo, P. (2014). Manual para la Formación y Capacitación en Mediación Comunitaria. Programa de las Naciones Unidas para el Desarrollo https://cutt.ly/fYhzw3P

Pastor, E. y Huertas, E. (2012). La mediación penitenciaria como método alternativo de resolución de conflictos entre internos en el ámbito penitenciario. Entramado, 8(2), 139-153. https://revistas.unilibre. edu.co/index.php/entramado/article/view/3433

Plata, O. (2012). Tres interpretaciones de la teoría hobbesiana del conflicto. Filosofía UIS, (1), 119-130. https://revistas.uis.edu.co/index.php/revistafilosofiauis/article/view/3162

Redorta, J. (2004). Cómo analizar los conflictos. La tipología de conflictos como herramienta de mediación. Editorial Paidós. 
Redorta, J. (2007). Entender el conflicto. La forma como herramienta. Editorial Paidós.

Ríos, J., Pascual, E., Etxebarria, X., Segovia, J., y Lozano, F. (2016). La mediación penal, penitenciaria yencuentros restaurativos. Experiencias para reducir el sufrimiento en el sistema penal. Universidad Pontificia Comillas.

Romero Miranda, L. (2019). Prisionización: estructura y dinámica del fenómeno en cárceles estatales del sistema penal chileno. URVIO. Revista Latinoamericana De Estudios De Seguridad, (24), 42-58. https:// doi.org/10.17141/urvio.24.2019.3791

Salvador, C., y García, E. (2010). Compromiso e inteligencia emocional en mediadores del Poder Judicial de Oaxaca, México. Diversitas: Perspectivas en Psicología, (2), 375-387. https://doi.org/10.26807/revpuce. voio.117

Villanueva, R. (2017). Reglas mínimas de las Naciones Unidas para el Tratamiento de los Reclusos. Reglas de Mandela. Oficina de las Naciones Unidas Contra la Droga y el Delito. https://cutt.ly/GYhzULc

\section{AUTORA}

Martha Vanesa Díaz Padilla. Maestra en Ciencias Penales por la Universidad Anáhuac del Norte y Licenciada en Psicología por UNAM. Presidenta del Instituto de Ciencias Psico, Jurídico, Sociales IPSIJUS.

Directora y Fundadora de la Asociación Mexicana en Psicología Jurídica y Derecho Penal AMPJyDP. Representante de México por la Organización Mundial de Mediación, con sede en Berlín. 